\title{
Serum NFL discriminates Parkinson disease from essential tremor and reflect motor and cognition severity
}

Yixian Huang ${ }^{1,2^{*}+}$, Caili Huang ${ }^{3+}$, Qilin Zhang ${ }^{1}$, Tingting Shen ${ }^{1}$ and Jiawei Sun ${ }^{1}$

\begin{abstract}
Objective: To investigate the diagnostic value of serum neurofilament light chain (NFL) for discriminating Parkinson disease (PD) from Essential tremor (ET) and healthy controls, and to evaluate its correlation with some clinical features of PD patients.

Methods: This cross-sectional study measured NFL levels with electrochemiluminescence immunoassay in serum of 146 PD patients, 82 ET patients and 60 age-matched healthy controls. We used multivariate regression analyses to examine whether NFL contributes to PD biomarkers. Disease severity were assessed by Unified Parkinson's Disease Rating Scale part III (UPDRS III), Hoehn \& Yahr (H-Y) stage and Mini-Mental State Examination (MMSE).

Results: Serum NFL levels were significantly higher in PD than in ET and healthy controls (16.6 $\pm 3.5,12.2 \pm 2.4$ and $11.8 \pm 2.4 \mathrm{pg} / \mathrm{mL}$, respectively, $p<0.001$ ). In patients with PD, serum NFL were markedly increased in patients with advanced $\mathrm{H}-\mathrm{Y}$ stage and patients with dementia (both $p<0.001$ ). The correlation analysis revealed that serum NFL was positively associated with UPDRS III score $(r=0.79, p<0.001)$ and $\mathrm{H}-Y$ stage $(r=0.86, p<0.001)$, and negatively correlated with MMSE scores $(r=-0.70, p<0.001)$. Further multivariate regression analyses showed that serum NFL was an independent contributor to motor symptom and cognition severity in PD patients (all $p<0.01$ ).
\end{abstract}

Conclusions: Serum NFL levels were markedly elevated may be a useful clinical biomarker for discriminating PD patients from ET and controls. Serum NFL may serve as a potential blood biomarker for motor and cognition severity of PD.

Keywords: Parkinson's disease, Neurofilament light chain, Essential tremor, Biomarker

\section{Background}

Historically, Parkinson's disease (PD) has been considered primarily as a motor disorder [1]. However, In addition to typical motor symptoms, cognitive impairment is one of the most disabling nonmotor features for PD patients and caregivers, and dementia eventually develops in a significant proportion of PD patients [2].

\footnotetext{
*Correspondence: huangyx88863552@163.com

†Yixian Huang and Caili Huang contributed equally to this work.

${ }^{1}$ Department of Neurology, The Second Affiliated Hospital of Soochow

University, NO. 1055 Sanxiang Road, Suzhou 215004, China

Full list of author information is available at the end of the article
}

Essential tremor (ET) is generally regarded as a benign movement disorder with single symptom, and has now been replaced by the view that it may be a heterogeneous neurodegenerative disease [3]. Most PD patients suffer from the tremor dominant type, which has the most symptomatic overlap with ET [4]. Classic ET is characterized by action tremor, which affects the upper limbs in at least $95 \%$ of patients. Some PD patients also show postural tremor. Therefore, the differential diagnosis of the two diseases is difficult in some cases. Up to now, no specific blood-biomarker have been identified for 
differentiating PD patients from ET. A promising candidate of such biomarkers is the neurofilament light chain (NFL).

NFL is a neuronal cytoskeletal protein, which is released into the CSF during neuroaxonal damage and has been shown to be elevated in several neurodegenerative disorders [5-9]. Recently, there are some conflicting reports on the blood level of NFL in PD patients [10]. There is emerging evidence that NFL levels in the blood/ CSF of PD patients may be significantly increased in comparison to healthy control subjects and are associated with motor impairment and cognitive decline in patients with PD [11, 12]. We hypothesize that serum NFL may be used to distinguish PD from ET and reflect the disease severity of PD. Therefore, our aim was to study the diagnostic value of serum NFL for differentiating PD from ET, and to evaluate its correlation with motor symptoms and cognitive impairment severity of PD patients.

\section{Materials and methods \\ Participants}

All participants were recruited from the Second Affiliated Hospital of Soochow University. This study included 288 participants: 146 patients with idiopatic PD, 82 patients with ET, and 60 healthy controls. All patients with PD met the following inclusion criteria: (1) age 40-80years old, Han Chinese; (2) All patients were diagnosed independently by two neurologists, according to the United Kingdom PD Society Brain Bank Criteria [13]. patient with atypical parkinsonism such as the multiple system atrophy, progressive supranuclear palsy, and vascular or secondary parkinsonism was excluded. (3) $>2$ years diagnosed with PD. (4) receiving stable doses of L-Dopa/carbidopa administered at least 3 times per day for at least 4 weeks before the initial screening visit.

Patients with ET met the diagnostic criteria by the Consensus Statement of the Movement Disorder Society on Tremor [14]. Additionally, 60 age- and sex-matched healthy controls with no history of any neurological or psychiatric diseases were recruited from their spouses and friends of patients with PD at the same period. All subjects were Chinese Han population and at least 8 years of formal education.

We received approval from the ethics committee of the Second Affiliated Hospital of Soochow University. All participants gave written informed consent before inclusion in this study.

\section{Motor symptom severity assessment}

We assessed motor symptom severity of the PD patients using the Hoehn \& Yahr (H-Y) stage and the Unified Parkinson's Disease Rating Scale part III (UPDRS III) score as previously literature described. Which was assessed only during "on" condition $[15,16]$.

We measured Tremor severity and functional effects of the ET patients using the Fahn-Tolosa-Marin Tremor Rating Scale (TRS) subscales A, B and C score as previously literature described [17]. Each subscale element is rated from 0 to 4 (none to severe tremor) giving a maximum score of 16, 36 and 32 for each subscale.

According to the Jankovic et al's criteria (1990) [18], based on the UPDRS, we subdivided PD patients into the tremor-dominant (TD) and postural instability/gait disorder (PIGD) subtypes.

\section{Diagnostic criteria for PD or ET with mild cognitive impairment and dementia}

$\mathrm{PD}$ with mild cognitive impairment (PD-MCI) and with dementia (PDD) were diagnosed with the diagnostic criteria of the Movement Disorder Society Task Force $[19,20]$. An MMSE score of 26 to 28 was used as a possible PD-MCI diagnostic feature as previously literature described $[9,21]$. An MMSE score of $\leq 25$ was used as a possible PDD diagnostic feature as previously literature described $[9,21]$. The diagnostic criteria of ET and control with cognitive impairment was consistent with those of PD. Structural MRI and routine laboratory tests were performed for all patients to exclude non-PDD causes of dementia.

\section{Measurement of NFL}

We collected $10 \mathrm{ml}$ peripheral blood from each participant between 8:00 and 9:00 AM prior to clinical assessment and following an overnight fast. Blood samples were centrifuged ( $2500 \mathrm{~g}$ for $15 \mathrm{~min}$ ) within $1 \mathrm{~h}$ of collection and then kept frozen at $-80^{\circ} \mathrm{C}$ until assay. Serum NFL concentrations were determined by researchers who were blinded to the clinical diagnosis, and serum samples were transferred onto the single molecule array (Simoa) NF-light Advantage Kit from Quanterix (Lexington, MA), as previously described [10].

\section{Statistical analyses}

Demographic and clinical variables between groups were compared using analysis of variance (ANOVA) for continuous variables, and chi-squared for categorical variables. Since serum NFL levels were normally distributed in in all three groups (Kolmogorove-Smirnov one-sample test, for PD group: $p=0.22$; for ET group: $p=0.14$ and for control group: $p=0.36$ ), the parametric tests were used. For variables that violated the assumptions of normality, the groups were compared with nonparametric Mann-Whitney $U$ test (for 2 groups) or Kruskal-Wallis test (for $>2$ groups). We compared NFL levels among the three groups using ANOVA, and 
Fisher's least significant difference (LSD) test was performed for Bonferroni post-hoc pairwise comparisons. Where there was a significance in ANOVA, the effect of sex, age, education, BMI and clinical variables was tested by adding these variables to the analysis model as covariates. To determine the diagnostic accuracy, a receiver operating characteristic (ROC) curve was constructed to determine the area under the curve (AUC) and the values of sensitivity and specificity with 95\% confidence interval $(\mathrm{CI})$, and Youden index was determined (sensitivity + specificity - 1.0) to find the optimal cutoff value. Relationships between variables were assessed with Pearson correlation and Spearman Rank correlation, where appropriate. Bonferroni corrections were applied to each test to adjust for multiple testing. A multivariate regression analyses was used to assess association of serum NFL with UPDRS III, H-Y and MMSE while adjusting for potentially confounding demographic and clinical variables, including sex, age, education, BMI, alcohol, age of onset and duration of disease. Effect sizes $(0.2=$ small effect, $0.5=$ medium effect, $0.8=$ large difference effect) were calculated using Cohen's $d$ method for the two-way comparisons and represented the mean difference, in standard deviation units, between the groups of interest. The SPSS Statistics (version 19.0, IBM Corp., Armonk, NY, USA) was utilized for all statistical analyses. All data are expressed as mean and standard deviation (mean $\pm \mathrm{SD}$ ). All $p$-values of $<0.05$ was considered significant.

\section{Results}

\section{Clinical characteristics of participants}

The study included 288 participants: 146 patients with PD, 82 patients with ET, and 60 were healthy controls. Demographic and clinical information and serum NFL concentrations of all participants are shown in Table 1 . The three groups were matched for age, sex and education. PD patients and ET patients were not significantly different in body mass index (BMI), age of onset and disease duration (all $p>0.05$ ). The TRS score of ET patients was $33.2 \pm 16.7$. The MMSE scores were significantly lower in patients with PD $(28.4 \pm 2.9)$ than in patients with ET $(29.4 \pm 1.4)$ and in healthy controls $(29.8 \pm 0.4)$ (both $p<0.01$; effect sizes $=0.44$ and 0.68 , respectively; Kruskal-Wallis test; Table 1). Table 1 shows the mean $\mathrm{H}-\mathrm{Y}$ stages $(2.5 \pm 1.0)$ and UPDRS part III scores $(23.2 \pm 8.9)$ during the "on" condition.

\section{Serum NFL concentrations in PD and ET}

To determine whether older age might influence serum NFL levels, we examined the correlation between the age of the subjects and NFL levels. Consistent with a previous report, higher serum NFL levels were associated with older age in healthy controls, patients with PD and patients with ET (control: $\mathrm{r}=0.30,95 \%$ CI 0.05-0.52, $p=0.02$; PD: $r=0.25,95 \%$ CI $0.07-0.42, p=0.002$; ET: $\mathrm{r}=0.30,95 \% \mathrm{CI} 0.13-0.46, p=0.006$; Pearson correlation analysis). The serum NFL concentrations were significantly higher in patients with PD $(16.6 \pm 3.5 \mathrm{pg} / \mathrm{ml})$ than

Table 1 Clinical characteristics and serum NFL levels of participants

\begin{tabular}{|c|c|c|c|c|}
\hline Characteristics & Controls $(n=60)$ & Patients with PD (n=146) & Patients with ET ( $n=82)$ & $P$ Value $^{\text {a }}$ \\
\hline $\operatorname{Sex}, M / F$ & $79 / 67$ & $34 / 26$ & $43 / 39$ & 0.88 \\
\hline age, $y$ & $60.5 \pm 11.3$ & $61.5 \pm 9.6$ & $60.6 \pm 8.8$ & 0.71 \\
\hline Education, y & $9.0 \pm 1.6$ & $9.0 \pm 1.7$ & $9.0 \pm 1.9$ & 0.92 \\
\hline BMI, $\mathrm{kg} / \mathrm{m}^{2}$ & $23.9 \pm 2.2$ & $23.7 \pm 1.8$ & $23.7 \pm 2.4$ & 0.5 \\
\hline Disease duration, $y$ & NA & $4.9 \pm 2.8$ & $10.6 \pm 2.3$ & $<0.01^{b}$ \\
\hline Age of onset, $y$ & NA & $56.7 \pm 8.7$ & $50.0 \pm 8.3$ & $<0.01^{\mathrm{b}}$ \\
\hline MMSE score & $29.8 \pm 0.4$ & $28.4 \pm 2.9$ & $29.4 \pm 1.4$ & $<0.01^{\mathrm{b}, \mathrm{c}}$ \\
\hline H-Y stages & NA & $2.5 \pm 1.0$ & NA & NA \\
\hline UPDRS part III scores & NA & $23.2 \pm 8.9$ & NA & NA \\
\hline TRS scores & NA & NA & $33.2 \pm 16.7$ & NA \\
\hline NFL, pg/ml & $11.8 \pm 2.4$ & $16.6 \pm 3.5$ & $12.2 \pm 2.4$ & $<0.01^{\mathrm{b}, \mathrm{c}}$ \\
\hline
\end{tabular}

Abbreviations: $B M I$ body mass index; $E T$ essential tremor; $H-Y$ Hoehn \& Yahr; MMSE Mini-mental state examination; NA not available; NFL neurofilament light chain; $P D$ Parkinson disease; UPDRS united Parkinson's disease rating scale

Values are expressed as the mean \pm SD

a Parameters were analyzed with analysis of variance using Bonferroni as post hoc test in the case of normal distribution of data. For variables that did not display a normal distribution, data were compared with the Kruskal-Wallis test for comparison of multiple groups, or Mann-Whitney $U$ test for comparison of 2 groups. Sex was analyzed using $\mathrm{X} 2$ test

${ }^{b}$ Differences were found between PD vs ET

${ }^{c}$ Differences were found between PD vs control 
in patients with ET $(12.2 \pm 2.4 \mathrm{pg} / \mathrm{ml})$ and in healthy controls $(11.8 \pm 2.4 \mathrm{pg} / \mathrm{ml})$ (both $p<0.01$; effect sizes $=1.47$ and 1.60, respectively; Table 1 and Fig. 1A). As shown in Table 2, PD patients were classified as having the TD and PIGD motor subtypes. There were no significant differences in demographic and NFL level variables in PD patients (all $p>0.05$ ). The ROC analysis showed that a serum NFL cutoff value of $13.75 \mathrm{pg} / \mathrm{ml}$ had a sensitivity of $76 \%$ and a specificity of $85 \%$ for distinguishing between PD and healthy controls, with an area under the curve of 0.869 (Fig. 1B). Because serum NFL was higher in patients with $\mathrm{PD}$ than in patients with $\mathrm{ET}(p<0.01$, Fig. 1A), the serum NFL cutoff value of $13.65 \mathrm{pg} / \mathrm{ml}$ had a $76.7 \%$ sensitivity and a $84.1 \%$ specificity for distinguishing between PD and ET, with an area under the curve of 0.854 (Fig. 1C).

\section{Serum NFL levels were associated with motor severity and cognitive status in PD}

Motor disorder is the most typical dysfunctionis of PD patients. In assessing the motor symptom severity of PD, we observed an rose in serum NFL levels with the increasing motor disorder as reflected by the $\mathrm{H}-\mathrm{Y}$ stage (Fig. 2A). The Specific serum NFL concentrations were $12.2 \pm 1.8,15.1 \pm 2.1,18.0 \pm 1.5,20.6 \pm 1.5$, and $25.1 \pm 1.5 \mathrm{pg} / \mathrm{ml}$ for $\mathrm{H}-\mathrm{Y}$ stage I through $\mathrm{V}(n=27$, $n=46, n=47, n=23, n=3)$, respectively ( $\mathrm{F}=104.1$, $p<0.001)$. Cognitive impairment is one of the most
A

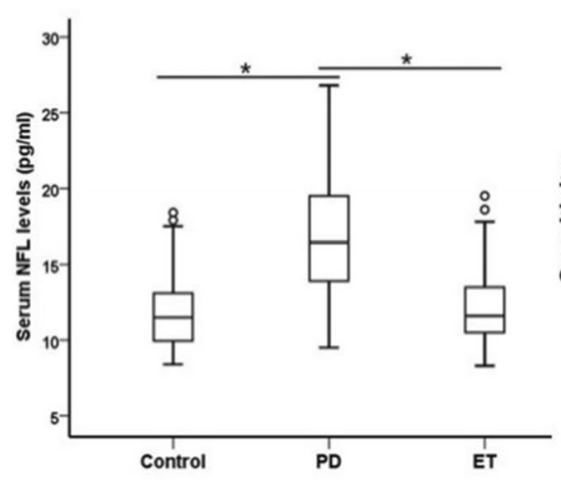

B. PD vs Control

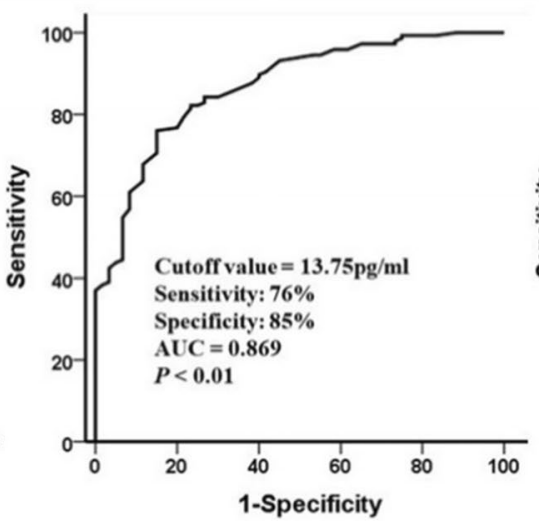

\section{PD vs ET}

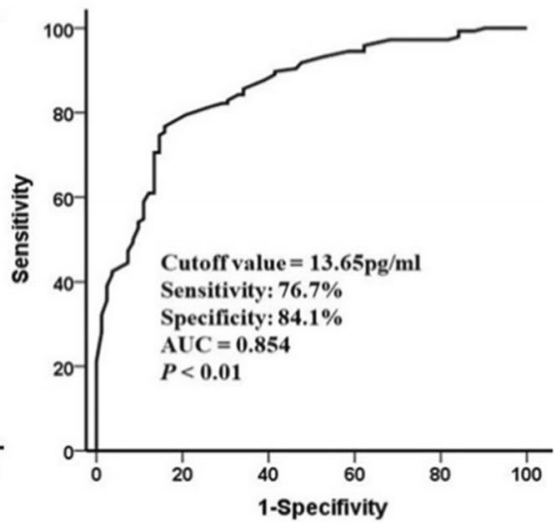

Fig. 1 Serum NFL highly discriminates PD from ET and controls. Serum NFL concentration in healthy controls, patients with PD and patients with ET and diagnostic accuracy. (A) Mean serum NFL level was significantly elevated in PD compared to ET and nonneurologic healthy controls. Mean levels were shown with $S D ;{ }^{*} p<0.01$. (B, C), Receiver operating characteristic curve analyses for differentiating between (B) patients with PD and age-matched healthy controls and (C) patients with PD and those with ET. AUC = area under the curve; $E T=$ Essential tremor; $N F L=$ neurofilament light chain; $\mathrm{PD}=$ Parkinson disease

Table 2 Comparison between TD-PD and PIGD-PD motor subtypes on demographic details and NFL variables

\begin{tabular}{|cccc}
\hline & \multicolumn{3}{c}{ PD } \\
\cline { 2 - 4 } & TD (n=71) & PIGD (n=75) & P Value \\
\hline Sex (M/F) & $34 / 37$ & $33 / 42$ & 0.638 \\
\hline Age (years) & $55.77 \pm 9.40$ & $57.48 \pm 7.90$ & 0.236 \\
\hline Education (years) & $9.13 \pm 1.93$ & $8.87 \pm 1.46$ & 0.357 \\
\hline NFL (pg/ml) & $16.43 \pm 3.23$ & $16.73 \pm 3.70$ & 0.604 \\
\hline
\end{tabular}

Note: Data are presented as the mean \pm standard deviation (Mean \pm SD)

Abbreviations: NFL neurofilament light chain; PD Parkinson disease; PIGD postural instability/gait disturbances dominant; TD tremor dominant

Parameters were analyzed with analysis of variance using Bonferroni as post hoc test in the case of normal distribution of data. For variables that did not display a normal distribution, data were compared between TD-PD and PIGD-PD groups by Mann-Whitney U test. Sex was analyzed using X2 test. 

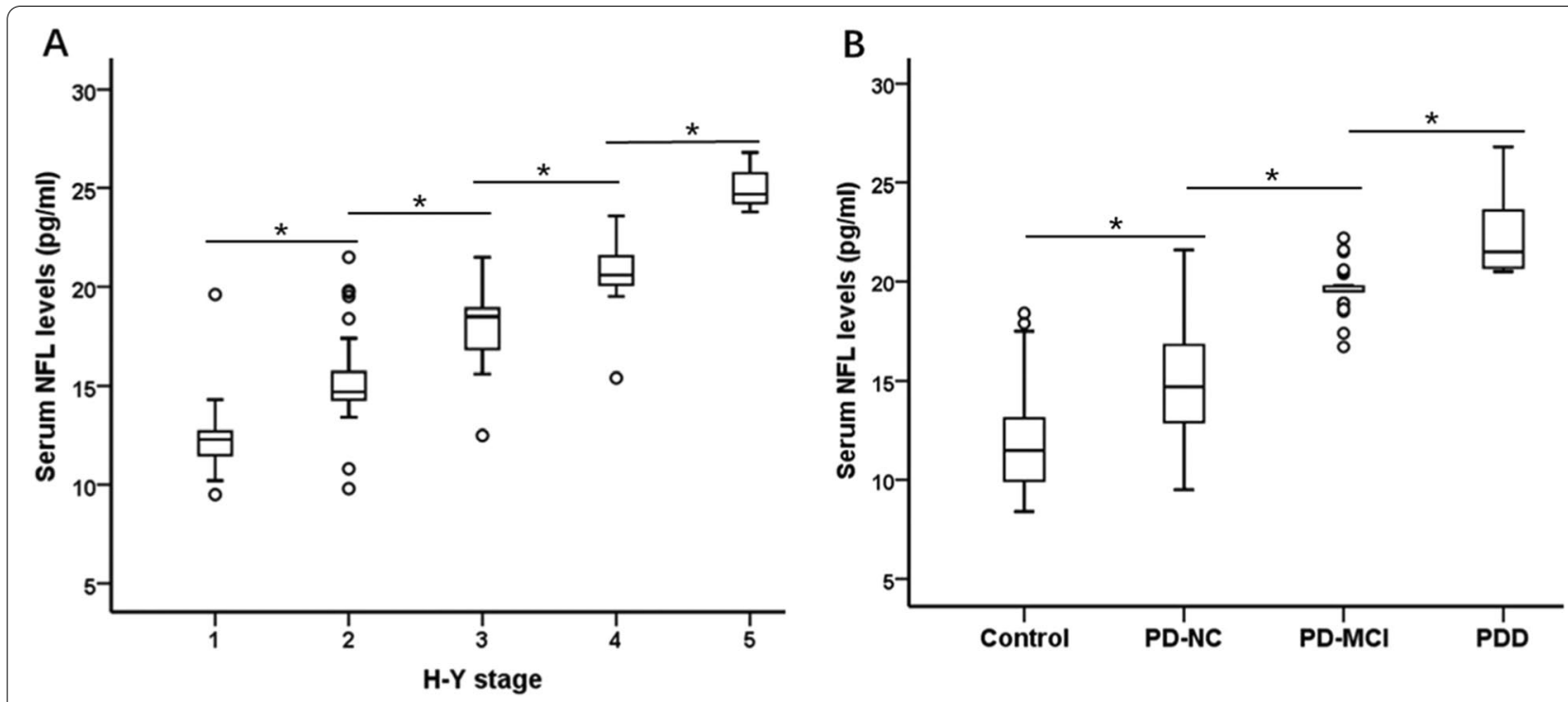

Fig. 2 Serum NFL in PD according to motor and cognition severity. (A) Mean serum NFL level was significantly elevated in patients with PD who displayed severe motor disorder based on the H-Y stage $(p<0.01)$. (B) Mean serum NFL level was markedly higher in patients with PDD compared to PD-MCl, PD-NC and control. Mean levels are shown with SD; ${ }^{*} p<0.01 . \mathrm{H}-\mathrm{Y}=\mathrm{Hoehn} \&$ Yahr; $\mathrm{MCl}=$ mild cognitive impairment; $\mathrm{NFL}=$ neurofilament light chain; $\mathrm{PD}=$ Parkinson disease; $\mathrm{PDD}=\mathrm{PD}$ with dementia; $\mathrm{PD}-\mathrm{NC}=\mathrm{PD}$ with normal cognition

disabling nonmotor features of PD. We then examined the relationship between serum NFL concentrations and various levels of cognitive function among PD patients and healthy controls. We observed that serum NFL significantly increased as the cognitive impairment severity increased (Fig. 2B). The specific NFL concentrations were $11.8 \pm 2.4,15.0 \pm 2.6,19.6 \pm 1.1$, and $22.2 \pm 1.9 \mathrm{pg} / \mathrm{ml}$ for the control, PD with normal cognition, PD-MCI, and PDD groups $(n=60, n=102, n=31, n=13)$, respectively $(\mathrm{F}=114.8, p<0.001)$.

In patients with $\mathrm{PD}$, correlation analysis showed that serum NFL levels were significantly positively correlated with motor symptom severity, measured with UPDRS III score and H-Y stage (UPDRS III score: $\mathrm{r}=0.79,95 \%$ CI 0.70-0.86, $p<0.001$; Bonferroni corrected $p<0.01$; Pearson correlation analysis; $\mathrm{H}-\mathrm{Y}$ stage: $\mathrm{r}=0.86,95 \% \mathrm{CI}$ $0.78-0.91, p<0.001$; Bonferroni corrected $\mathrm{p}<0.01$; Spearman correlation analysis) (Fig. 3A, Fig. 2A). At the same time, serum NFL levels were significantly negatively correlated with MMSE scores in PD patients (MMSE scores: $r=-0.70,95 \% \mathrm{CI}-0.63$ to $-0.77, p<0.001$; Bonferroni corrected $\mathrm{p}<0.01$; Pearson correlation analysis) (Fig. 3B).

Further multivariate regression analysis showed that serum NFL was an independent contributor to motor symptom and cognition decline severity in $\mathrm{PD}$ patients $(\mathrm{H}-\mathrm{Y}$ stage: $\mathrm{t}=8.75, \quad \mathrm{VIF}=2.93, \quad p<0.001$; UPDRS III score: $\mathrm{t}=3.17$, VIF $=2.92, P=0.002$; MMSE score: $\mathrm{t}=-4.81, \mathrm{VIF}=1.73, p<0.001$ ), while adjusting for potentially confounding demographic and clinical variables, including age, sex, education, BMI, age of onset, and disease duration. Our data has been normalized and there was no multicollinearity.

\section{Discussion}

In this study, we aimed to analyze whether serum NFL levels could be used as a biomarker for discriminating PD from ET and controls and reflect the motor and cognition severity of PD. For this purpose, we measured NFL concentrations in the serum of patients with PD, ET and age-matched healthy controls. Our results demonstrated that serum NFL levels were significantly increased in PD than ET and controls and discriminated PD from ET and controls with high accuracy levels. Correlation analysis showed that serum NFL was positively associated with UPDRS III score and H-Y stage, and negatively correlated with MMSE scores. Further multivariate regression analysis confirmed that serum NFL was an independent contributor to motor symptom severity and cognition decline in patients with PD, while adjusting for potential confounders, including age, sex, education, BMI, age of onset, and disease duration. Altogether, these findings suggested that increased serum NFL may serve as a surrogate biomarker of motor and cognition severity in $\mathrm{PD}$ and discriminated PD from ET and controls.

Abnormal neuronal cytoskeletal proteins is one of the major pathological mechanisms of several neurological disorders $[9,22]$. NFL is abundant in axons, thus release of NFL likely occurs after neuroaxonal damage 

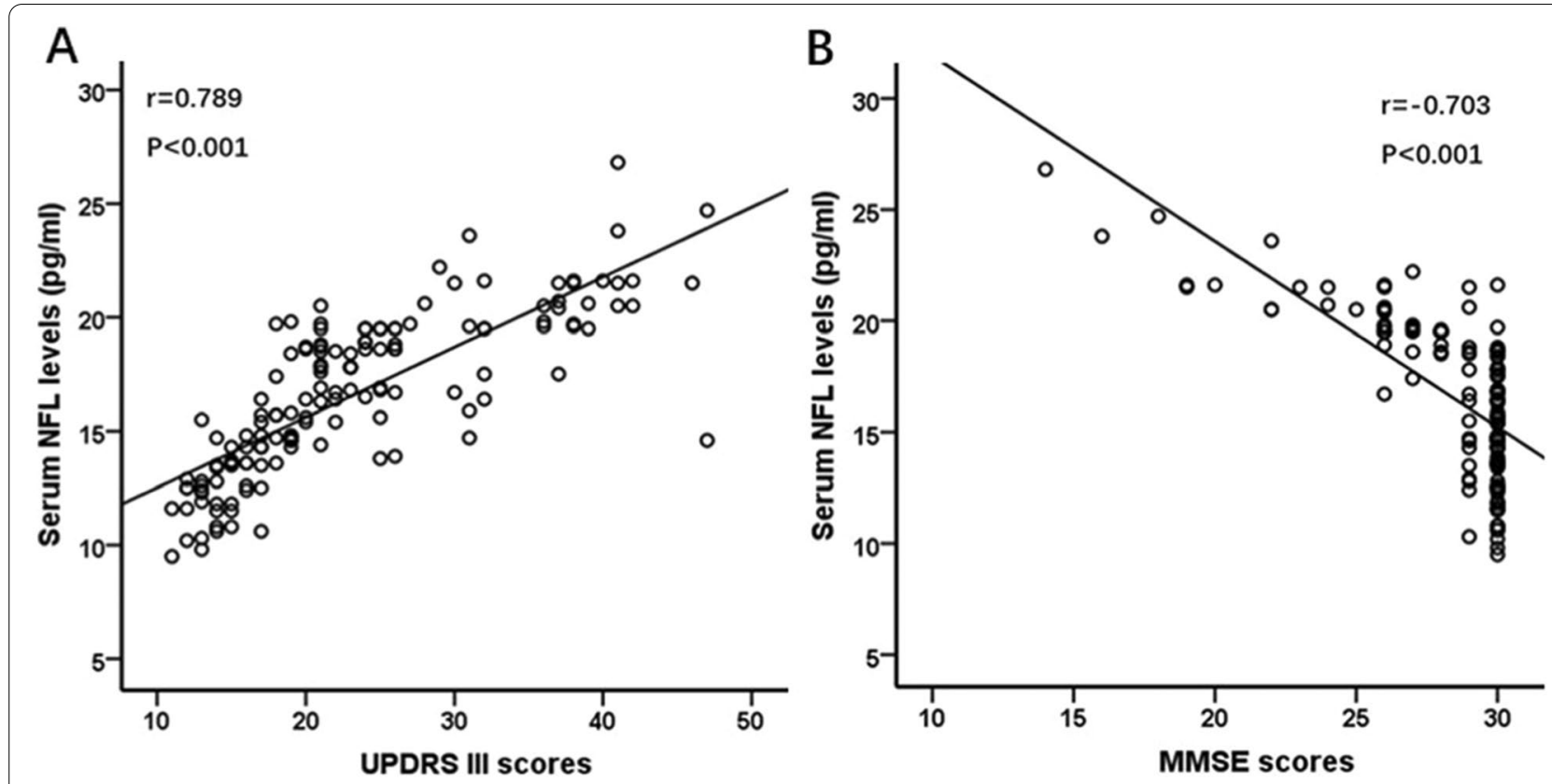

Fig. 3 Serum NFL was associated with motor and cognition severity in PD. Correlation analysis showed that (A) serum NFL levels were significantly positively correlated with UPDRS III scores $(p<0.001)$, (B) serum NFL levels were markedly negatively correlated with MMSE scores $(p<0.001)$. MMSE = Mini-Mental State Examination; NFL = neurofilament light chain; PD = Parkinson disease; UPDRS III = Unified Parkinson's Disease Rating Scale part III

with subsequent release into blood [23, 24]. Recent several studies showed that NFL has become a potential biomarker in PD $[9,25]$. Some authors have found NFL levels were elevated in PD patients compared to healthy controls $[9,11]$, many others have observed no differences between these two groups of subjects [12, 26], but the conclusions were based either on a smaller number of PD patients or involved patients with less advanced PD. So far, the correlation between NFL levels and PD patients remains unclear. Clinically, most PD patients suffer from the tremor dominant type with a most symptomatic overlap with ET [4]. ET is a most prevalent agerelated neurological diseases. The precise pathogenesis remains to be elucidated. Recent studies using western blot analysis found no difference in NFL protein expression between the ET patients and controls [27]. Our findings demonstrated that serum NFL levels were markedly elevated in PD compared to ET or controls and discriminated PD from ET and control with high accuracy. The ROC analysis showed that the serum NFL had a sensitivity $(76.7 \%$ ) and a $84.1 \%$ specificity (AUC 0.85 ) for distinguishing between patients with PD and ET. These results suggested that serum NFL may be a useful clinical biomarker for differentiating PD patients from ET and healthy controls.

In the present study, the three groups of subjects were matched for age, sex and education. we found that higher serum NFL levels were associated with older age in three groups, regardless of disease status. This result was consistent with those previously reported [28]. Nonetheless, the reason for this association between age and serum NFL levels remains to be elucidated. Presumably, it could be due to age-related bloodstream clearance decreases with aging or brain hypometabolism, leading to some degree of axonal degeneration and NFL release into bloodstream [23-25].

Motor disorder is the most typical dysfunctions of PD patients. When the various levels of motor ability among patients with PD were considered, serum NFL level showed a positive correlation with motor symptom severity, measured with H-Y stage and UPDRS III score. Interestingly, we observed an rose in serum NFL levels with the increasing motor disorder as reflected by the $\mathrm{H}-\mathrm{Y}$ stage I through V. Cognitive decline is one of the most disturbing nonmotor features of PD. When the various levels of cognitive ability among patients with PD and controls were considered, serum NFL showed a negative correlation with MMSE score, a clinical measure of global cognitive function in PD. It is interesting that serum NFL levels rose with increasing cognitive decline as reflected by lowest levels MMSE score in participants with PDD through highest MMSE score in healthy controls. These findings were consistent with a recent study published by Lin et al. [9], they found a positive 
correlation between plasma NFL levels and PD severity assessed in H-Y stage, UPDRS III score and MMSE score. Altogether, our results suggested that, in addition to the diagnostic value of serum NFL levels for differentiating PD from ET and controls, serum NFL might be a biomarker for motor symptom and cognition severity in PD patients.

The current study has some limitations. First, the study design was cross-sectional, which limits causal inference between NFL and clinical features of PD. Second, although we enrolled patients with PD and ET had been diagnosed for more than 2 years, they were diagnosed based on clinical criteria and not a pathological examination, especially for PDD patients. Third, we did not collect some more important clinical data, such as family history, coffee intake, levodopa equivalent dose (LED) calculation, hypertension, diabetes, coronary heart disease and other information, which may also affect the clinical symptoms or serum NFL in PD patients. Fourth, our clinical trial population was entirely han chinese and lacked ethnic diversity. Fifth, the relatively small sample size of each group is a concern. We performed a longitudinal follow-up study in conjunction with neuroimages comparison of PD, ET and controls, which may serve as supplemental evidence for future clinical use of serum NFL as a diagnostic biomarker.

\section{Conclusion}

In conclusion, our findings suggest that markedly elevated serum NFL levels may be a useful clinical biomarker for differentiating PD patients from ET and controls. Furthermore, we found a correlation of serum NFL with disease severity in terms of motor and cognitive performance in PD patients. As such, elevated serum NFL may serve as a potential blood biomarker of motor and cognition severity in PD. A future clinical trial with a larger longitudinal follow-up studies that incorporate other biomarkers such as $\alpha$-synulcein and neuroimages are needed to validate whether blood NFL may be used to predict PD progression.

\section{Abbreviations \\ AUC: Area under the curve; BMI: Body mass index; Cl: Confidence interval; ET: Essential tremor; H-Y: Hoehn \& Yahr stage; LED: Levodopa equivalent dose; MCI: Mild cognitive impairment; MMSE: Mini-Mental State Examination; NFL: Neurofilament light chain; PD: Parkinson disease; PDD: PD with dementia; ROC: Receiver operating characteristic curve; Simoa: Single molecule array; UPDRS III: Unified Parkinson's Disease Rating Scale part III.}

\section{Acknowledgments}

Not applicable.

\section{Authors' contributions}

Study concept and design: Yixian Huang, Caili Huang, Qilin Zhang. Acquisition of data: Yixian Huang, Caili Huang, Qilin Zhang, Jiawei Sun, Tingting Shen. Analysis and interpretation of data: Yixian Huang, Qilin Zhang. Drafting and
Critical revision of the manuscript for important intellectual content: Yixian Huang. Statistical analysis: Yixian Huang, Qilin Zhang. Study supervision: Yixian Huang. All authors reviewed and contributed to the manuscript and approved the final version.

\section{Funding}

This work was supported by the National Natural Science Foundation of China (NO.81200970), the Suzhou Sci \& Tech Program Grant (SYSD2018098) and Changzhou High-Level Medical Talents Training Project (NO.2016CZBJ023).

\section{Availability of data and materials}

The datasets used and/or analyzed during the current study are available from the corresponding author on reasonable request.

\section{Declarations}

\section{Ethics approval and consent to participate}

The studies involving human participants were reviewed and approved by the Human Ethics Committees of the Second Affiliated Hospital of Soochow University. Informed consent was obtained for experimentation with human subjects. Every individual was informed about the aims of the study and provided written consent before participation. Written Informed consent was obtained from healthy controls and from the legal guardian/next of kin for Parkinson's Disease patients. All methods were carried out in accordance with relevant guidelines and regulations/Declaration of Helsinki.

\section{Consent for publication}

Not applicable.

\section{Competing interests}

The authors declare that they have no conflict of interest.

\section{Author details}

${ }^{1}$ Department of Neurology, The Second Affiliated Hospital of Soochow University, NO. 1055 Sanxiang Road, Suzhou 215004, China. ${ }^{2}$ Department of Psychiatry, Johns Hopkins University School of Medicine, Baltimore, MD 21205, USA. ${ }^{3}$ Department of hematology, The Second Affiliated Hospital of Soochow University, NO. 1055 Sanxiang Road, Suzhou 215004, China.

Received: 22 September 2021 Accepted: 11 January 2022 Published online: 27 January 2022

\section{References}

1. Kalia LV, Lang AE. Parkinson's disease. Lancet. 2015;386:896-912.

2. Hely MA, Reid WG, Adena MA, Halliday GM, Morris JG. The Sydney multicenter study of Parkinson's disease: the inevitability of dementia at 20 years. Mov Disord. 2008;23:837-44.

3. Bonuccelli U. Essential tremor is a neurodegenerative disease. J Neural Transm (Vienna). 2012;119:1383-7.

4. Algarni M, Fasano A. The overlap between essential tremor and Parkinson disease. Parkinsonism Relat Disord. 2018;46(Suppl 1):S101-4.

5. Lu CH, Macdonald-Wallis C, Gray E, et al. Neurofilament light chain: a prognostic biomarker in amyotrophic lateral sclerosis. Neurology. 2015;84:2247-57.

6. Piehl F, Kockum I, Khademi M, et al. Plasma neurofilament light chain levels in patients with MS switching from injectable therapies to fingolimod. Mult Scler. 2018;24:1046-54.

7. Sandelius $\AA$, Zetterberg H, Blennow K, et al. Plasma neurofilament light chain concentration in the inherited peripheral neuropathies. Neurology. 2018;90:e518-24.

8. Quiroz YT, Zetterberg H, Reiman EM, et al. Plasma neurofilament light chain in the presenilin 1 E280A autosomal dominant Alzheimer's disease kindred: a cross-sectional and longitudinal cohort study. Lancet Neurol. 2020;19(6):513-21.

9. Lin $\mathrm{CH}$, Li CH, Yang KC, et al. Blood NfL: a biomarker for disease severity and progression in Parkinson disease. Neurology. 2019;93(11):e1104-11. 
10. Marques TM, van Rumund A, Oeckl P, et al. Serum NFL discriminates Parkinson disease from atypical parkinsonisms. Neurology. 2019;92(13):e1479-86.

11. Oosterveld LP, Verberk IMW, Majbour NK, et al. CSF or serum neurofilament light added to a-Synuclein panel discriminates Parkinson's from controls. Mov Disord. 2020;35(2):288-95.

12. Parnetti L, Gaetani L, Eusebi P, et al. CSF and blood biomarkers for Parkinson's disease. Lancet Neurol. 2019;18(6):573-86.

13. Hughes AJ, Daniel SE, Kilford L, Lees AJ. Accuracy of clinical diagnosis of idiopathic Parkinson's disease: a clinic-pathological study of 100 cases. J Neurol Neurosurg Psychiatry. 1992;55:181-4.

14. Deuschl G, Bain P, Brin M. Consensus statement of the Movement Disorder Society on tremor. Ad Hoc Scientific Committee Mov Disord. 1998;13(Suppl 3):2-23.

15. Hoehn MM, Yahr MD. Parkinsonism: onset, progression and mortality. Neurology. 1967;17:427-42.

16. Fahn S, Elton R. Unified Parkinson's Disease Rating Scale. In: Fahn S, Marsden CD, Caine DB, Goldstein M, editors. Recent developments in Parkinson's disease, vol 2. Florham Park: Macmillan Health Care information; 1987. p. 153-63. 293-304.

17. Fahn, S.; Tolosa, E.; Marin, C. Clinical Rating Scale for Tremor. In Parkinson's Disease and Movement Disorders; Jankovic, J., Tolosa, E., Eds.; Urban \& Schwarzenberg: Baltimore-Münich, WA, USA, 1988; pp. 225-234.

18. Jankovic J, McDermott M, Carter J, et al. Variable expression of Parkinson's disease: a base-line analysis of the DATATOP cohort. Parkinson Study Group Neurol. 1990;40:1529-2134.

19. Emre $M$, Aarsland D, Brown R, et al. Clinical diagnostic criteria for dementia associated with Parkinson's disease. Mov Disord. 2007;22:1689-837.

20. Litvan I, Goldman JG, Troster Al, et al. Diagnostic criteria for mild cognitive impairment in Parkinson's disease: Movement Disorder Society task force guidelines. Mov Disord. 2012;27:349-56.

21. Hoops S, Nazem S, Siderowf AD, Duda SX, Stern MB, Weintraub D. Validity of the MoCA and MMSE in the detection of $\mathrm{MCl}$ and dementia in Parkinson disease. Neurology. 2009;73:1738-45.

22. Lawton MP, Brody EM. Assessment of older people: self-maintaining and instrumental activities of daily living. Gerontologist. 1969;9:179-86.

23. Khalil $M$, Teunissen $C E$, Otto $M$, et al. Neurofilaments as biomarkers in neurological disorders. Nat Rev Neurol. 2018;14(10):577-89.

24. Gaetani L, Blennow K, Calabresi P, Di Filippo M, Parnetti L, Zetterberg H. Neurofilament light chain as a biomarker in neurological disorders. J Neurol Neurosurg Psychiatry. 2019;90(8):870-81.

25. Lerche $S$, Wurster I, Röben B, et al. CSF NFL in a longitudinally assessed PD cohort: age effects and cognitive trajectories. Mov Disord. 2020. https:// doi.org/10.1002/mds.28056, https://doi.org/10.1002/mds.28056.

26. Wang SY, Chen W, Xu W, et al. Neurofilament light chain in cerebrospinal fluid and blood as a biomarker for neurodegenerative diseases: a systematic review and Meta-analysis. J Alzheimers Dis. 2019;72(4):1353-61.

27. Louis ED, Ma K, Babij R, et al. Neurofilament protein levels: quantitative analysis in essential tremor cerebellar cortex. Neurosci Lett. 2012;518(1):49-54.

28. Jin M, Cao L, Dai YP. Role of Neurofilament light chain as a potential biomarker for Alzheimer's disease: a correlative Meta-analysis. Front Aging Neurosci. 2019;11:254.

\section{Publisher's Note}

Springer Nature remains neutral with regard to jurisdictional claims in published maps and institutional affiliations.

Ready to submit your research? Choose BMC and benefit from:

- fast, convenient online submission

- thorough peer review by experienced researchers in your field

- rapid publication on acceptance

- support for research data, including large and complex data types

- gold Open Access which fosters wider collaboration and increased citations

- maximum visibility for your research: over 100M website views per year

At BMC, research is always in progress.

Learn more biomedcentral.com/submissions 